\title{
Atypical neurofibromatosis type 1 with unilateral limb hypertrophy mimicking overgrowth syndrome
}

\author{
K. Tripolszki, ${ }^{1}$ K. Farkas, ${ }^{2}$ A. Sulák, ${ }^{1}$ G. Szolnoky, ${ }^{3}$ B. Duga, ${ }^{4}$ B. Melegh, ${ }^{4}$ R. G. Knox, ${ }^{5}$ \\ V. E. R. Parker, ${ }^{5}$ R. K. Semple, ${ }^{5}$ L. Kemény, ${ }^{2,3}$ M. Széll ${ }^{1,2}$ and N. Nagy ${ }^{1,2,3}$ \\ ${ }^{1}$ Department of Medical Genetics, ${ }^{2}$ MTA-SZTE Dermatological Research Group and ${ }^{3}$ Department of Dermatology and Allergology, University of Szeged, \\ Szeged, Hungary; ${ }^{4}$ Department of Medical Genetics, University of Pécs, Pécs, Hungary; and ${ }^{5}$ Metabolic Research Laboratories, Institute of Metabolic \\ Science, Addenbrooke's Hospital Cambridge, Cambridge, UK
}

doi:10.1111/ced.13154

\section{Summary}

\begin{abstract}
Neurofibromatosis type 1 (NF1; OMIM 162200), a dominantly inherited multitumor syndrome, results from mutations in the Neurofibromin 1 (NF1) gene. We present the case of a Hungarian woman with the clinical phenotype of NF1 over her whole body and the clinical features of unilateral overgrowth involving her entire left leg. This unusual phenotype suggested either the atypical form of NF1 or the coexistence of NF1 and overgrowth syndrome. Direct sequencing of the genomic DNA isolated from peripheral blood revealed a novel frameshift mutation (c.5727insT, p.V1909fsX1912) in the NF1 gene. Next-generation sequencing of 50 oncogenes and tumour suppressor genes, performed on the genomic DNAs isolated from tissue samples and peripheral blood, detected only wild-type sequences. Based on these results, we concluded that the patient is affected by an unusual phenotype of NF1, and that the observed unilateral overgrowth of the left leg might be a rare consequence of the identified c.5727insT mutation.
\end{abstract}

Neurofibromatosis type 1 (NF1; OMIM 162200) is a rare monogenic disease with autosomal dominant inheritance, caused by mutations in the Neurofibromin 1 (NF1) gene. 1 The clinical features of NF1 involve pigmentary changes such as café-au-lait macules and axillary freckling, development of cutaneous fibromatous tumours, and development of hamartomas of the iris known as Lisch nodules. ${ }^{1}$ Besides these symptoms, central nervous system and skeletal abnormalities (scoliosis, pseudoarthrosis of the tibia, macrocephaly and short stature) can occur. ${ }^{2,3}$ Frequently reported vascular symptoms include vascular dysplasia with cerebral, gastrointestinal and/or renal involvement, and renovascular hypertension. ${ }^{2,3}$

NF1 may be caused by many different loss-offunction mutations of the NF1 gene. ${ }^{1}$ In about $5 \%$ of

Correspondence: Dr Nikoletta Nagy, Department of Medical Genetics, University of Szeged, 4 Somogyi utca, H-6720 Szeged, Hungary

E-mail: nikoletta.nagy@gmail.com

Conflict of interest: the authors declare that they have no conflicts of interest.

Accepted for publication 28 June 2016 patients with NF1, microdeletions of the NF1 gene and/or the surrounding genomic region are responsible for the disease. ${ }^{4}$ In patients with NF1 microdeletion, somatic overgrowth has been frequently observed. ${ }^{5}$ Mosaic overgrowth affecting a well-defined region of the body have only been rarely reported in NF1.

We report the case of a Hungarian woman presenting with the classic clinical features of NF1 and with unusual unilateral overgrowth of her left leg. The symptoms of NF1 were visible over her whole body, while the overgrowth was present only in her left leg. Our aim was to identify the underlying genetic abnormality of this unusual case and determine whether the patient is affected by two independent diseases or whether the symptoms of the left leg are an unusual manifestation of NF1.

\section{Report}

A 52-year-old Hungarian woman presented with an unusual phenotype, exhibiting the clinical features of 
NF1. The typical skin symptoms of NF1 were present in the patient, including neurofibromas and cafe-aulait macules on her body (Fig. 1a,b), and axillary freckling. Ophthalmological examination determined the presence of Lisch nodules. Imaging studies did not find any indication of central nervous system malignancies. Skeletal abnormalities such as scoliosis, tibial pseudoarthrosis, short stature and macrocephaly, were not present. Based on the clinical symptoms, the diagnosis of NF1 was established.

However, in addition to the aforementioned clinical features, the patient was also noted to have hypertrophy of the left leg, resulting in significant differences in the circumference and length of the legs (Fig. 1c,d). This abnormality of the left leg was already present at birth. Imaging studies verified unilateral osteohypertrophy affecting the left leg. The patient was not aware of any relevant chronic diseases or other family members with NF1 or with overgrowth syndromes (Fig. 1e).

Following ethics approved by the internal review board of the University of Szeged (Szeged, Hungary), genetic studies were carried out, conducted according to the principles of the Declaration of Helsinki. Written informed consent was obtained from all participants. Tissue biopsy of the left leg and blood sample were taken from the patient, and genomic DNA was isolated (BioRobot EZ1 DSP Workstation; QIAGEN, Hilden, Germany). Her family members were unwilling to provide material for genetic investigation.
To confirm NF1, the coding regions and flanking introns of the NF1 gene were amplified and sequenced. Direct sequencing of genomic DNA isolated from the peripheral blood sample revealed a novel frameshift mutation (c.5727insT, p.V1909fsX1912) in exon 39 of the NF1 gene (Fig. 2). The patient carried the mutation in heterozygous form (Fig. 2a), while unrelated controls $(n=50)$ carried the wild-type sequence (Fig. 2b).

To verify overgrowth syndrome, the coding regions and flanking introns of the PIK3CA gene were amplified and sequenced. As traditional sequencing of genomic DNA isolated from the peripheral blood and from the tissue sample of the left leg did not identify any putative causative variant of the PIK3CA gene, mutational hotspots were screened using an in-house PCRbased restriction fragment assay. ${ }^{6}$ As these also did not identify any overgrowth-causing mutation, nextgeneration sequencing of approximately 2800 mutations from 50 oncogenes and tumour suppressor genes recorded in the Catalogue of Somatic Mutations in Cancer (COSMIC) was performed (Ion AmpliSeq ${ }^{{ }^{\mathrm{TM}_{\mathrm{V}}}}$ Cancer Hotspot Panel v2; Thermo Fisher Scientific, Waltham, MA, USA), but also did not identify any putative causative variant that could be responsible for the overgrowth of the left leg.

This patient presented with the clinical features of both NF1 and left leg overgrowth. Such an atypical clinical form of NF1 has not been reported previously. However, the phenomenon of 'vascular neurofibromatosis phenotype' and the relatively frequent
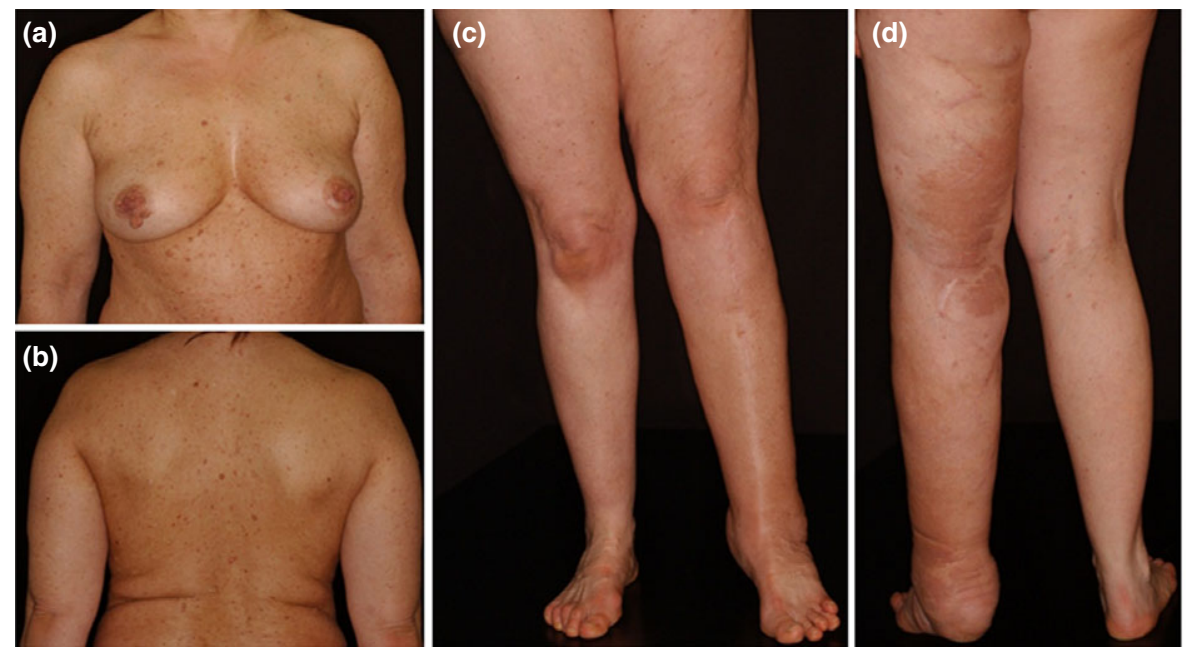

(e)
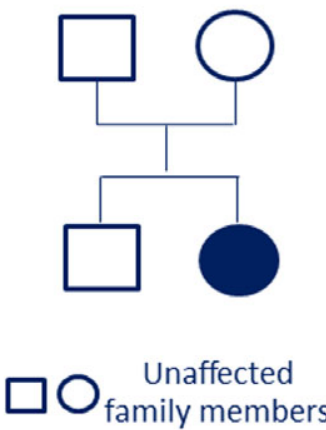

Investigated patient

Figure 1 An unusual phenotype of neurofibromatosis (NF)1 in a 52-year-old Hungarian woman. The patient presented with the clinical phenotype of NF1 over her whole body, including (a) her chest and (b) back. (c,d) Unilateral osteohypertrophy affected the left leg. (e) The rest of the patient's family were clinically asymptomatic. 
(a)
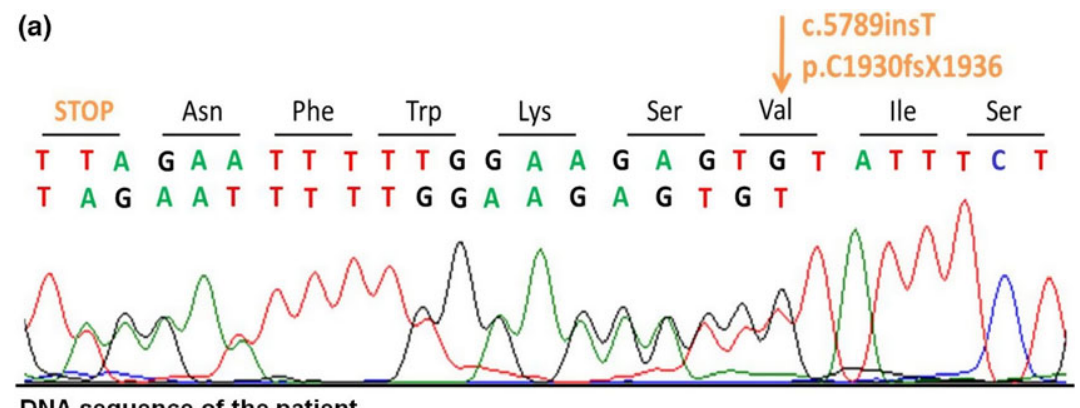

DNA sequence of the patient

(b)
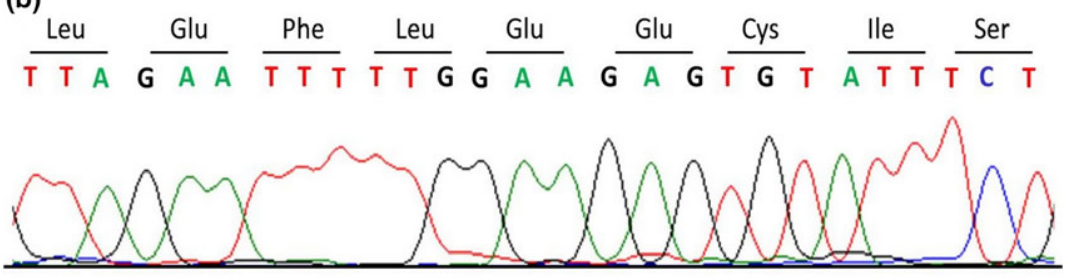

DNA sequence of the controls

Figure 2 (a,b) Direct sequencing of the NF1 gene. Direct sequencing revealed a novel single-nucleotide insertion resulting in a frameshift and the formation of premature termination codon (c.5727insT, p.V1909fsX1912). (a) The patient carried the mutation in heterozygous form. (b) Unrelated, healthy controls carried only wild-type sequences.

association of NF1 with vascular dysplasia have been frequently reported in the literature. ${ }^{7}$ NF1 can also be accompanied by skeletal abnormalities, such as sphenoid wing dysplasia, macrocephaly, scoliosis, vertebral disc dysplasia, pseudoarthrosis of tibia and short stature. $^{8,9}$ Therefore, it was important to distinguish whether the patient was affected by two independent rare diseases or the symptoms of the left leg were the results of the atypical vascular and skeletal manifestations of NF1.

Our results demonstrated a novel heterozygous single-nucleotide insertion in the NF1 gene, leading to a frameshift and the formation of a premature termination codon (c.5727insT, p.V1909fsX1912). Because this mutation was present in the genomic DNA isolated from the peripheral blood of the patient, we suggest that this sequence change is a germline mutation. Considering that there is no other clinically affected member in the patient's family and that NF1 exhibits autosomal dominant inheritance, we hypothesize that this is a de novo mutation of the NF1 gene. The genotype-phenotype correlation is generally poor in NF1, with the exception that patients with large deletions in the NF1 gene tend to have severe phenotypes. $^{10}$ NF1 mutations usually result in loss of tumour suppressor function by disrupting the NF1 protein's ability to maintain the proto-oncogene RAS in an inactive form. ${ }^{11}$ We suggest that this novel NF1 mutation is the causative mutation for the development of NF1, and that it is highly possible that the presence of the unilateral limb hypertrophy is also the consequence of this mutation.

In conclusion, we report a patient with both the clinical features of NF1 and overgrowth of the left leg. To our knowledge, our study is the first to clearly elucidate the genetic background of such a complex case, and further confirms the causative role of the somatic mutations of the NF1 gene in the development of overgrowth.

\section{Acknowledgements}

This research was supported by the European Social Fund in the framework of TÁMOP-4.2.2/B-10/1/ KONV-2010-0012 and TÁMOP-4.2.2.A-11/1/KONV2012-0035; by Wellcome Trust (grants WT097721 and WT098498 to VERP and RKS) and the Medical Research Council (MRC_MC_UU_12012/5); and by the UK National Institute Health Research (NIHR) Cambridge Biomedical Research Centre and Rare Diseases Translational Research Collaboration (to RGK).

\section{Learning points}

- The case reported in this study was presented with the clinical features of both NF1 and left leg overgrowth. 
- The unusual clinical phenotype including the left leg overgrowth is the atypical manifestation of NF1.

- The patient carries a disease-causing germline mutation of the NF1 gene.

- The patient does not carry any somatic mutation of PIK3CA or other genes.

- Our study elucidates the genetic background of the investigated case with unusal phenotype, and confirms that the observed left leg overgrowth is the consequence of NF1.

\section{References}

1 Huson SM. The neurofibromatosis: classification, clinical features and genetic counselling. In: Neurofibromatoses (Kaufmann D, ed). Basel: Karger, 2008; 1-20.

2 Mukonoweshuro W, Griffiths PD, Blaser S. Neurofibromatosis type 1: the role of neuroradiology. Neuropediatrics 1999; 30: 111-19.
3 Stevenson DA, Birch PH, Friedman JM et al. Descriptive analysis of tibial pseudarthrosis in patients with neurofibromatosis 1. Am J Med Genet 1999; 84: 413-19.

4 Pasmant E, Sabbagh A, Spurlock G et al. NF1 microdeletions in neurofibromatosis type 1: from genotype to phenotype. Hum Mutat 2010; 31: 1506-18.

5 Spiegel M, Oexle K, Horn D et al. Childhood overgrowth in patients with common NF1 microdeletions. Eur J Hum Genet 2005; 13: 883-8.

6 Keppler-Noreuil KM, Sapp JC, Lindhurst MJ, et al. Clinical delineation and natural history of the PIK3CA-related overgrowth spectrum. Am J Med Genet A 2014; 164A: 1713-33.

7 Reubi F. Neurofibromatose et lesions vasculaires. Schweiz Med Wochenschr 1945; 75: 463-5.

8 Ferner RE, Huson SM, Thomas N et al. Guidelines for the diagnosis and management of individuals with neurofibromatosis 1. J Med Genet 2007; 44: 81-8.

9 Williams VC, Lucas J, Babcock MA et al. Neurofibromatosis type 1 revisited. Pediatrics 2009; 123: 124-33.

10 Heim RA, Silverman LM, Farber RA et al. Screening for truncated NF1 proteins. Nat Genet 1994; 8: 218-19.

11 Yohay KH. The genetic and molecular pathogenesis of NF1 and NF2. Semin Pediatr Neurol 2006; 13: 21-6. 